\title{
Some Remarks on the RRR Linkage
}

\author{
J.M. Selig \\ Faculty of Business \\ London South Bank University, U.K.
}




\section{Introduction - Dual Quaternions/Study Quadric Reminder}

Represent rigid displacements as dual quaternions,

$$
\left(a_{0}+a_{1} i+a_{2} j+a_{3} k\right)+\varepsilon\left(b_{0}+b_{1} i+b_{2} j+b_{3} k\right)
$$

Think of $a_{0}, a_{1}, \ldots, b_{3}$ as homogeneous coordinates in a $\mathbb{P}^{7}$.

Rigid-displacements satisfy the equation,

$$
a_{0} b_{0}+a_{1} b_{1}+a_{2} b_{2}+a_{3} b_{3}=0
$$

degree 2 equation, variety called the Study quadric. Points on the quadric in 1-to-1 correspondence with group elements except for those with $a_{0}=a_{1}=a_{2}=a_{3}=0$. 


\section{The General $3 R$ Linkage}

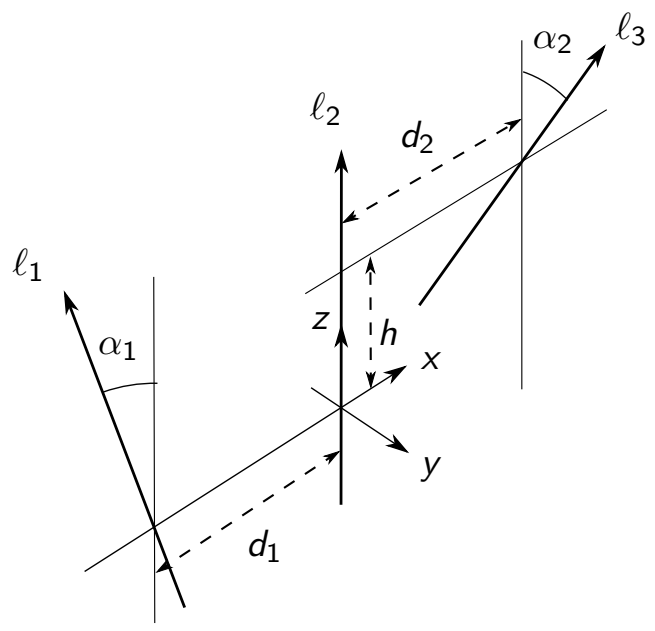

Look at displacements generated by end-effector of general 3R linkage. Here $\ell_{i}$ is the $i$ th joint axis. 


\section{Parametric Representation}

Use dual quaternions to express rigid displacements. In general,

$$
\begin{aligned}
\left(a_{0}+a_{1} i+a_{2} j+a_{3} k\right)+\varepsilon\left(b_{0}+b_{1} i+b_{2} j+b_{3} k\right)= \\
\left(c_{1}+s_{1} \ell_{1}\right)\left(c_{2}+s_{2} \ell_{2}\right)\left(c_{3}+s_{1} \ell_{3}\right)
\end{aligned}
$$

Here $c_{i}, s_{i}$ are the parameters, can think of them as Cosine and Sine of joint half-angles. The joint axes are given by lines,

$$
\ell=\left(\omega_{x} i+\omega_{y} j+\omega_{z} k\right)+\varepsilon\left(v_{x} i+v_{y} j+v_{z} k\right)
$$

wth $\omega_{i}$ and $v_{j}$ the components of the direction along the joint axis and its moment vector respectively. 


\section{Parametric Representation}

Can write this in matrix form,

$$
\left(\begin{array}{l}
a_{0} \\
a_{1} \\
a_{2} \\
a_{3} \\
b_{0} \\
b_{1} \\
b_{2} \\
b_{3}
\end{array}\right)=M\left(\begin{array}{c}
c_{1} c_{2} c_{3} \\
s_{1} c_{2} c_{3} \\
c_{1} s_{2} c_{3} \\
c_{1} c_{2} s_{3} \\
s_{1} s_{2} s_{3} \\
-c_{1} s_{2} s_{3} \\
s_{1} c_{2} s_{3} \\
-s_{1} s_{2} c_{3}
\end{array}\right)
$$

The matrix $M$ has columns,

$$
M=\left(1\left|\ell_{1}\right| \ell_{2}\left|\ell_{3}\right| \ell_{1} \ell_{2} \ell_{3}\left|-\ell_{2} \ell_{3}\right| \ell_{1} \ell_{3} \mid-\ell_{1} \ell_{2}\right)
$$




\section{The Matrix}

Where,

$$
1=(1,0,0,0,0,0,0,0)^{T}
$$

and

$$
\ell=\left(0, \omega_{x}, \omega_{y}, \omega_{z}, 0, v_{x}, v_{y}, v_{z}\right)^{T}
$$

and so forth.

When $M$ is non-singular this is just a projective transformation of the standard Segre variety $\mathbb{P}^{1} \times \mathbb{P}^{1} \times \mathbb{P}^{1}$. That is just a (projective) change of coordinates. 


\section{Nine Quadrics - Implicit Representation}

If we write,

$$
\begin{aligned}
& X_{0}=c_{1} c_{2} c_{3}, \quad X_{1}=s_{1} c_{2} c_{3}, \quad X_{2}=c_{1} s_{2} c_{3}, \quad X_{3}=c_{1} c_{2} s_{3}, \\
& Y_{0}=s_{1} s_{2} s_{3}, \quad Y_{1}=-c_{1} s_{2} s_{3}, \quad Y_{2}=s_{1} c_{2} s_{3}, \quad Y_{3}=-s_{1} s_{2} c_{3} .
\end{aligned}
$$

then the standard Segre variety lies on 9 quadrics,

$$
\begin{array}{lll}
Q_{1}: X_{0} Y_{1}+X_{2} X_{3}=0, & Q_{4}: X_{1} X_{0}+Y_{2} Y_{3}=0, \\
Q_{2}: X_{0} Y_{2}-X_{1} X_{3}=0, & Q_{5}: X_{2} Y_{0}-Y_{1} Y_{3}=0, \\
Q_{3}: X_{0} Y_{3}+X_{2} X_{3}=0, & Q_{6}: X_{3} Y_{0}+Y_{1} Y_{2}=0 .
\end{array}
$$

$$
\begin{aligned}
& Q_{7}: X_{0} Y_{0}+X_{1} Y_{1}=0 \\
& Q_{8}: X_{0} Y_{0}-X_{2} Y_{2}=0, \\
& Q_{9}: X_{0} Y_{0}+X_{3} Y_{3}=0 .
\end{aligned}
$$

Could use $M^{-1}$ to write 9 quadrics in the coordinates $a_{0}, a_{1}, \ldots, b_{3}$. 


\section{Application - Line Symmetric 6R Mechanism}

Can the end-effector of such a $3 R$ linkage undergo a line-symmetric motion?

If yes, then could join the end-effectors of two identical $3 R$ linkages to get a mobile $6 R$ closed-loop mechanism. The coupler bar will trace the line symmetric motion.

Line symmetric motions lie in the intersection of the Study quadric with certain 5-planes. Intersecting the Segre variety with a general $\mathbb{P}^{5}$ gives a curve in general. Hence the answer to the question above is: Yes. So line-symmetric $6 R$ mechanisms are mobile.

Moreover, the degree of the curve will be 6 , since it is well known that the Segre variety has degree six. 


\section{Genus of the Curve}

In general this configuration curve must be elliptic (genus 1 ),

- A degree 6 curve in a $\mathbb{P}^{7}$ can be either rational or elliptic. Project from points until you get a cubic in $\mathbb{P}^{2}$ which is known to be rational or elliptic.

- Cannot be rational since it lies in 9 quadrics. A rational curve of degree six $n \mathbb{P}^{7}$ can only lie in 8 quadrics.

Many other easy consequences for closed loop mechanisms and 6R robots. 


\section{The Determinant of $M$}

Using parameterisation above, can compute the determinant of $M$.

$$
\begin{aligned}
\operatorname{det}(M)= & -h^{4} \sin ^{4} \alpha_{1} \sin ^{4} \alpha_{2} \\
& -2 h^{2} \sin ^{2} \alpha_{1} \sin ^{2} \alpha_{2}\left(d_{1}^{2} \sin ^{2} \alpha_{2}+d_{2}^{2} \sin ^{2} \alpha_{1}\right) \\
& -\left(d_{1}^{2} \sin ^{2} \alpha_{2}-d_{2}^{2} \sin ^{2} \alpha_{1}\right)^{2} .
\end{aligned}
$$

Careful analysis shows just 3 cases when $\operatorname{det}(M)=0$, 


\section{Result due to Josef Schicho}

The determinant of $M$ is zero if and only if one of the following conditions hold,

- All joint axes are parallel, $\sin \alpha_{1}=\sin \alpha_{2}=0$. Planar linkage.

- All joint axes are concurrent, $d_{1}=d_{2}=h=0$. Spherical linkage.

- Design parameters satisfy, $h=0$ and $\frac{d_{1}}{\sin \alpha_{1}}=\frac{d_{2}}{\sin \alpha_{2}}$. The Bennett conditions - call this $3 R$ a Bennett linkage. 


\section{Variety Generated by the Bennett Linkage}

Can show that the variety of displacements generated by the end-effector of such a Bennett linkage is the projection of the Segre variety from two point in the variety to a $\mathbb{P}^{5}$.

"Explains" mobility of the Bennett closed-loop $4 R$ mechanism: Rotation about the 4th joint of the Bennett mechanism corresponds to a line in the Study quadric. The only lines in $\mathbb{P}^{1} \times \mathbb{P}^{1} \times \mathbb{P}^{1}$ are the generator lines - rotations about the first three joints. However, twisted cubic curves in the Segre variety which meet the two projection centres will project to lines.

In fact possible to show that variety generated by the Bennett linkage is the complete intersection of a 5-plane with the Study quadric and one other quadric. So a variety of degree 4 . 


\section{Applications}

The coupler of a line-symmetric Bennett-Bennett mechanism traces an elliptic quartic curve in general - the intersection of two quadrics in a $\mathbb{P}^{3}$.

Can find the general number of postures for a $6 R$ serial robot with a Bennett linkage for its first three (or last 3 ) joints.

Bennett-3R wrist: 4 postures.

Bennett-Bennett: 8 postures.

Bennett-General 3R: 12 postures.

Several other easy results too. 


\section{Conclusions}

- Classical algebraic geometry provides simple but powerful tool to analyse the kinematics of robots and mechanisms. 


\section{Conclusions}

- Classical algebraic geometry provides simple but powerful tool to analyse the kinematics of robots and mechanisms.

- Hope to study mechanisms formed from several Bennett linkages. 


\section{Conclusions}

- Classical algebraic geometry provides simple but powerful tool to analyse the kinematics of robots and mechanisms.

- Hope to study mechanisms formed from several Bennett linkages.

THANK YOU 